\title{
Superfluid state of magnetoexcitons in double layer graphene structures
}

\author{
D. V. Fil, L. Yu. Kravchenko \\ Institute for Single Crystals National Academy of Sciences of Ukraine, \\ Lenin av. 60, 61001 Kharkov, Ukraine
}

\begin{abstract}
The possibility of realization of a superfluid state of bound electron-hole pairs (magnetoexcitons) with spatially separated components in a graphene double layer structure (two graphene layers separated by a dielectric layer) subjected by a strong perpendicular to the layers magnetic field is analyzed. We show that the superfluid state of magnetoexcitons may emerge only under certain imbalance of filling factors of the layers. The imbalance can be created by an electrostatic field (external gate voltage). The spectrum of elementary excitations is found and the dependence of the Berezinskii-KosterlitzThouless transition temperature on the interlayer distance is obtained. The advantages of use graphene double layer systems instead of double quantum well GaAs heterostructures are discussed.
\end{abstract}

Keywords: graphene, bilayer system, indirect excitons, interlayer phase coherence PACS: 1.35.Ji, 73.21.-b, 73.63.-b

\section{INTRODUCTION}

It is believed that excitons may demonstrate superfluid behavior. In bilayer systems superfluid excitons consisting of an electron from one layer and a hole from the other layer behave as superconductive ones. Indeed, having separate contacts in each layer one can use excitons for a nondissipative transmission of an electrical current from the source to the load (Fig. 11). The effect can be realized in double quantum wells in GaAs heterostuctures [1]. If such a system is subjected by a strong perpendicular to the layers magnetic field and the total filling factor of the Landau levels $v=1$, electrons that occupy quantum states in the zeroth Landau level in one layer couple with holes (empty states in the zeroth Landau level) in the other layer. Such pairs, called magnetoexcitons, are stable one, in difference with optically excited indirect excitons in double quantum wells [2, 3]. Stable excitons can emerge also in bilayers made of $n$-type and $p$-type two-dimensional conductors [4, 5]. In the latter systems the nesting of the Fermi surfaces of electrons and holes is required for the Bardin-Cooper-Schrieffer (BCS) pairing of $n$ and $p$ carriers.

The discovery of graphene [6, 7] has risen the idea of use double layer graphene systems for the realization of the exciton superconductivity. This question was already investigated in a number of papers [8, 9, 10, 11, 12].

Graphene can be considered as a semiconductor with zero band gap. Electron energy spectrum of graphene contains two Dirac points that separate the electron and the hole subband. In a bilayer structure the Fermi levels of the layers can be adjusted independently by the gate voltage. If the Fermi level is in the conduction band of one layer and is in the valence band of the other layer we have a $n-p$ bilayer. The electron-hole symmetry near the Dirac points ensures perfect nesting between the electron and the hole Fermi surfaces. Such a situation was considered in [8, 9, 10]. While the estimates in which the screening effects are neglected [8, 9] yield very high BCS temperature (hundreds of Kelvins), the screening may reduce the critical temperature down to 6 orders [10].

In [11, 12] the superfluid transition in the rarefied gas of magnetoexcitons was considered. Rarefied means that the number of magnetoexcitons is much smaller than the number of states in the Landau level. Since the critical temperature in $2 \mathrm{D}$ is proportional to the concentration of the carriers, the rarefied gas of magnetoexcitons cannot demonstrate record critical parameters. The aim of this paper is to analyze the case of high magnetoexciton density in bilayer graphene systems. Here we do not consider the effect of screening. In quantum Hall systems screening is expected to be small due to finite gaps between Landau levels. 


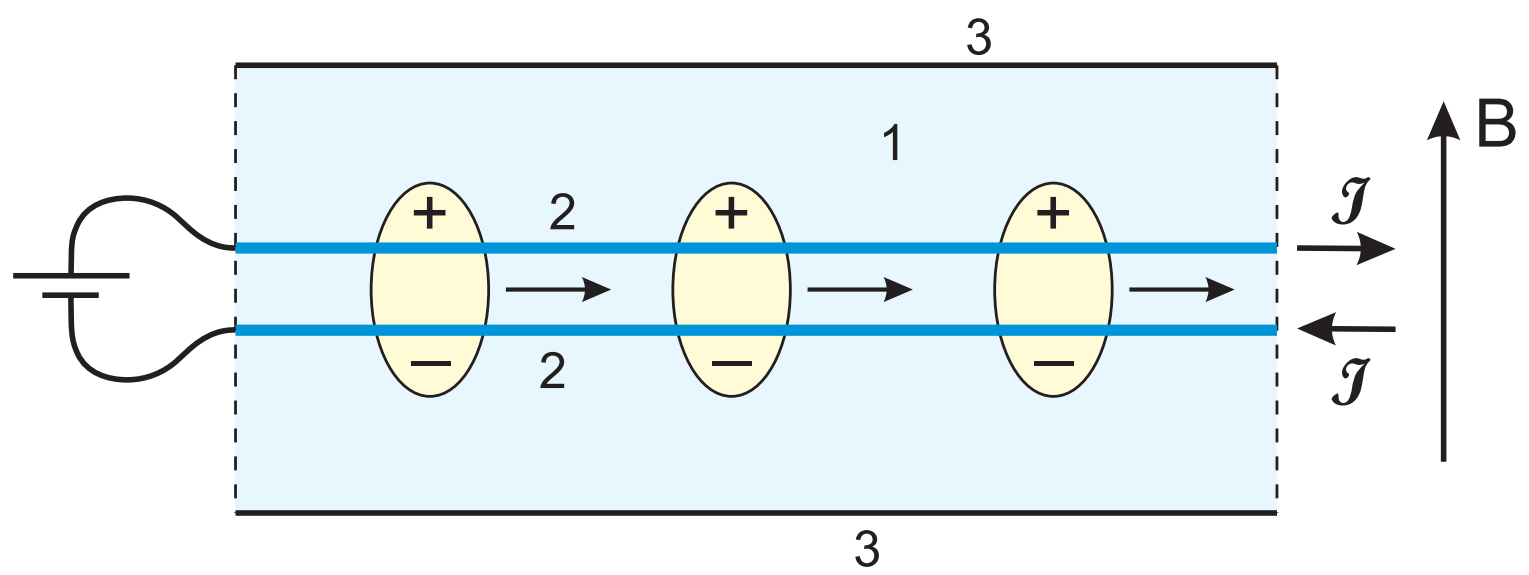

FIGURE 1. Bilayer graphene system (a dielectric matrix (1) with two embedded graphene layers (2) inside a capacitor (3)) that transmits the current by magnetoexcitons.

\section{INTERLAYER PHASE COHERENCE IN A DOUBLE LAYER GRAPHENE SYSTEM}

In conventional quantum Hall bilayers the case of high magnetoexciton density is realized at zero imbalance of filling factors of the layers $\left(v_{1}=v_{2}=1 / 2\right)$. The system at zero or moderate imbalance of filling factors can be regarded as an easy-plane quantum ferromagnet [13]. Here we extend the approach of Ref. [13] to a graphene bilayer system.

The Landau level energies and eigenfunctions for graphene are given by the equation [14]

$$
-i \hbar v_{F}\left(\begin{array}{cc}
0 & \frac{\partial}{\partial x}+\frac{\alpha x}{\ell^{2}}-i \alpha \frac{\partial}{\partial y} \\
\frac{\partial}{\partial x}-\frac{\alpha x}{\ell^{2}}+i \alpha \frac{\partial}{\partial y} & 0
\end{array}\right)\left(\begin{array}{l}
\Psi_{A} \\
\Psi_{B}
\end{array}\right)=E\left(\begin{array}{l}
\Psi_{A} \\
\Psi_{B}
\end{array}\right) .
$$

Here $v_{F} \approx 10^{6} \mathrm{~m} / \mathrm{s}$ is the Fermi velocity (for the Dirac spectrum $v_{F}$ is the matter parameter independent of the Fermi energy), $\ell=\sqrt{\hbar c / e B}$ is the magnetic length, $\alpha= \pm 1$ is the valley index (that corresponds to the carriers with the pseudo-momentum close to the $\mathbf{K}=(2 \pi / 3 a, 2 \pi / 3 \sqrt{3} a)$ and $\mathbf{K}^{\prime}=(2 \pi / 3 a,-2 \pi / 3 \sqrt{3} a)$ Dirac points, correspondingly), the spinor index $A(B)$ corresponds to the $A(B)$ sublattice of graphene. The solution of Eq. (1) yields the energies of the Landau levels in graphene

$$
E_{0}=0, \quad E_{ \pm N}= \pm \frac{\hbar v_{F}}{\ell} \sqrt{2 N}
$$

and the corresponding eigenfunctions $\Psi_{N, \alpha}$

$$
\begin{gathered}
\Psi_{0,-1}(x, y)=\frac{e^{-i k y}}{\pi^{1 / 4} \sqrt{\ell L_{y}}} e^{-\frac{(x-X)^{2}}{2 \ell^{2}}}\left(\begin{array}{c}
1 \\
0
\end{array}\right), \quad \Psi_{0,+1}(x, y)=\frac{e^{-i k y}}{\pi^{1 / 4} \sqrt{\ell L_{y}}} e^{-\frac{(x-X)^{2}}{2 \ell^{2}}}\left(\begin{array}{c}
0 \\
1
\end{array}\right), \\
\Psi_{ \pm N,-1}(x, y)=\frac{e^{-i k y}}{\pi^{1 / 4} \sqrt{\ell L_{y}} \sqrt{2^{N+1} N !}} e^{-\frac{(x-X)^{2}}{2 \ell^{2}}}\left(\begin{array}{c}
\mp H_{N}\left(\frac{x-X}{\ell}\right) \\
i \sqrt{2 N} H_{N-1}\left(\frac{x-X}{\ell}\right)
\end{array}\right), \\
\Psi_{ \pm N,+1}(x, y)=\frac{e^{-i k y}}{\pi^{1 / 4} \sqrt{\ell L_{y}} \sqrt{2^{N+1} N !}} e^{-\frac{(x-X)^{2}}{2 \ell^{2}}}\left(\begin{array}{c}
i \sqrt{2 N} H_{N-1}\left(\frac{x-X}{\ell}\right) \\
\mp H_{N}\left(\frac{x-X}{\ell}\right)^{\prime}
\end{array}\right) .
\end{gathered}
$$

Here $X=k \ell^{2}$ is the guiding center of the orbit, $H_{N}(x)$ is the Hermite polynomial, and $L_{y}$ is the size of the system in $y$ direction. Approximation (1) is valid for $\left|E_{N}\right| \ll 3 t$, where $3 t \approx 8 \mathrm{eV}$ is the half-width of the energy band of graphene (in the tight-binding approximation with the nearest-neighbor hopping energy $t$ ). We do not take into account the Zeeman splitting because it is much smaller than the energy distance between the Landau levels.

Due to the spin and valley degeneracy the total number of quantum states in the Landau level is $4 S / 2 \pi \ell^{2}(S$ is the area of the system). We define the filling factor of the Landau level as $v=2 \pi \ell^{2} n_{f}$, where $n_{f}$ is the concentration of occupied states. A completely filled level in graphene has the filling factor $v=4$. In undoped graphene the negative levels are completely filled, the positive levels are empty and the zeroth level has the filling factor $v=2$. 
The electron-hole pairing occurs between the carriers that belong to a partially filled Landau level (we will call it the active level). In particular, the pairing of electrons and holes belonging to the zeroth Landau level may take place. The many-body wave function that describes the state with such a pairing can be written analogously to [13]

$$
|\Psi\rangle=\prod_{X} \prod_{\beta}\left(\cos \frac{\theta_{\beta}}{2} a_{1, X \beta}^{+}+e^{i \varphi_{\beta}} \sin \frac{\theta_{\beta}}{2} a_{2, X \beta}^{+}\right)|0\rangle .
$$

Here $\beta=(\alpha, \sigma)$ are the sets of valley and spin quantum numbers (below we will notate them by digits $\beta=1,2,3,4$ ), $a_{i, k \beta}^{+}$is the operator of creation of an electron in the $i$-th layer in the active Landau level, $\varphi_{\beta}$ is the phase of the order parameter for the electron-hole pairing $\Delta_{\beta}=\left\langle\Psi\left|a_{1, \beta}^{+} a_{2, \beta}\right| \Psi\right\rangle=(1 / 2) \sin \theta_{\beta} e^{i \varphi_{\beta}}$, the parameter $\theta_{\beta}$ is connected with the filling factors of the component $\beta$ by the relation $v_{1(2), \beta}=\left(1 \pm \cos \theta_{\beta}\right) / 2$. The vacuum state $|0\rangle$ is the state with empty zeroth (active) and higher Landau levels in both layers.

Note that the function (5) can be presented in another form

$$
|\Psi\rangle=\prod_{X} \prod_{\beta}\left(\cos \frac{\theta_{\beta}}{2}+e^{i \varphi_{\beta}} \sin \frac{\theta_{\beta}}{2} a_{2, X \beta}^{+} h_{1, X \beta}^{+}\right)|v a c\rangle,
$$

where $h_{i, X \beta}^{+}$is the hole creation operator, and the vacuum state $|v a c\rangle$ is the state, where the zeroth Landau level in the layer 2 is empty, while in the layer 1 this level is completely filled. From (6) it becomes clear that the function (5) is just the analog of the BCS wave function. The state (5) is usually called the state with a spontaneous interlayer phase coherence.

We imply that the Coulomb energy $E_{c}=e^{2} / \varepsilon \ell$ ( $\varepsilon$ is the dielectric constant of the matrix in which the graphene layers are embedded) is much smaller than the energy distance between the active and the nearest passive (completely filled or completely empty) Landau level and take into account only the Coulomb interaction between electrons in the active level. Below we consider the case of $N=0$ active level. The Coulomb interaction has the form

$$
H_{C}=\frac{1}{2 S} \sum_{i, i^{\prime}} \sum_{\mathbf{q}} V_{i, i^{\prime}}(q) \hat{\rho}_{i}(\mathbf{q}) \hat{\rho}_{i^{\prime}}(-\mathbf{q})
$$

where $V_{i, i^{\prime}}(q)=\left(2 \pi e^{2} / \varepsilon q\right) \exp \left(-q d\left|i-i^{\prime}\right|\right)$ is the Fourier component of the Coulomb potential, $d$ is the interlayer distance, and

$$
\hat{\rho}_{i}(\mathbf{q})=\sum_{\beta} \sum_{k} a_{i, k+q_{y} / 2, \beta}^{+} a_{i, k-q_{y} / 2, \beta} \exp \left(-i q_{x} k \ell^{2}-\frac{q^{2} \ell^{2}}{4}\right)
$$

is the Fourier component of the electron density.

We consider the bilayer system situated inside the capacitor that creates an electrostatic field normal to the graphene layers. Varying the electrostatic field one can change the imbalance of filling factors of the layers. The energy of the system in the state (5) reads as

$$
E=\frac{S}{2 \pi \ell^{2}}\left(W\left(\sum_{\beta} \tilde{v}_{\beta}\right)^{2}-\mathscr{J}_{0}\left(1+\sum_{\beta} \tilde{v}_{\beta}^{2}\right)-\mathscr{J}_{1}\left(1-\sum_{\beta} \tilde{v}_{\beta}^{2}\right)-e V \sum_{\beta} \tilde{v}_{\beta}\right),
$$

where

$$
W=\frac{e^{2} d}{\varepsilon \ell^{2}}, \quad \mathscr{J}_{0}=\sqrt{\frac{\pi}{2}} \frac{e^{2}}{\varepsilon \ell}, \quad \mathscr{J}_{1}=\sqrt{\frac{\pi}{2}} \frac{e^{2}}{\varepsilon \ell} \exp \left(\frac{d^{2}}{2 \ell^{2}}\right) \operatorname{erfc}\left(\frac{d}{\sqrt{2} \ell}\right)
$$

are the energy constants that describe the direct interlayer interaction, the exchange intralayer interaction and the exchange interlayer interaction ( $\operatorname{erfc}(x)$ is the complementary error function). In (9) $V$ is the external gate voltage caused by the capacitor, and $\tilde{v}_{\beta}=\left(v_{1, \beta}-v_{2, \beta}\right) / 2$ is the filling factor imbalance for the component $\beta\left(\left|\tilde{v}_{\beta}\right| \leq 1 / 2\right)$. In (9) the interaction of electrons with the positively charged background is included. The result (9) can also be obtained in the standard mean-field approach [15].

The interaction constants satisfy the inequalities $\mathscr{J}_{0}>\mathscr{J}_{1}$ and $W-\mathscr{J}_{0}+\mathscr{J}_{1}>0$. One finds that at $V=0$ the minimum of (9) corresponds to $\tilde{v}_{1}=\tilde{v}_{2}=1 / 2, \tilde{v}_{3}=\tilde{v}_{4}=-1 / 2$. At such $\tilde{v}_{\beta}$ all the order parameters $\Delta_{\beta}$ are equal to zero and there is no electron-hole pairing. If the gate voltage is in the range

$$
\mathscr{J}_{0}-\mathscr{J}_{1}<e V<2 W-\mathscr{J}_{0}+\mathscr{J}_{1}
$$


the minimum is reached for

$$
\tilde{v}_{1}=\tilde{v}_{2}=\frac{1}{2}, \quad \tilde{v}_{3}=\frac{e V-W}{2\left(W-\mathscr{J}_{0}+\mathscr{J}_{1}\right)}, \quad \tilde{v}_{4}=-\frac{1}{2},
$$

and $\Delta_{3} \neq 0$.

For

$$
2 W+\mathscr{J}_{0}-\mathscr{J}_{1} \leq e V \leq 4 W-\mathscr{J}_{0}+\mathscr{J}_{1}
$$

the energy minimum corresponds to

$$
\tilde{v}_{1}=\tilde{v}_{2}=\tilde{v}_{3}=\frac{1}{2}, \quad \tilde{v}_{4}=\frac{e V-3 W}{2\left(W-\mathscr{J}_{0}+\mathscr{J}_{1}\right)},
$$

and $\Delta_{4} \neq 0$. Thus we conclude that an imbalance of filling factors is required for the electron-hole pairing and only electrons and holes with one $\beta$ are involved in the pairing. In the special cases $e V=W$ and $e V=3 W$ the imbalance of the active component has zero value, and the order parameter for the electron-hole pairing reaches the highest value.

The difference between the case of a quantum Hall bilayer in GaAs with the total filling factor $v=1$ and the case of the graphene bilayer system is the following. There is only one component in the first case and an imbalance of that component increases the direct interaction energy. In the second case positive imbalance of some components can be compensated be negative imbalance of the other components. The latter situation is similar to one that takes place in $v=2$ quantum Hall bilayers [16].

\section{CRITICAL TEMPERATURE}

The state with moving superfluid pairs is described by the order parameter with a spatially dependent phase. The many-body wave function of the state with a constant superfluid magnetoexciton current in $x$ direction reads as

$$
|\Psi\rangle=\prod_{X}\left(\cos \frac{\theta_{0}}{2} a_{1, X \beta}^{+}+e^{i Q X} \sin \frac{\theta_{0}}{2} a_{2, X \beta}^{+}\right)|0\rangle
$$

(the only part that corresponds to the active component is displayed). The part of energy that depends on $\theta_{0}$ and $Q$ is equal to

$$
E_{\mathrm{mf}}=-\frac{e \tilde{V} S \cos \theta_{0}}{2 \pi \ell^{2}}+\frac{S}{4 \pi \ell^{2}}\left(\frac{W-\mathscr{J}_{0}}{2} \cos ^{2} \theta_{0}-F_{D}(Q) \sin ^{2} \theta_{0}\right),
$$

where

$$
F_{D}(q)=\frac{e^{2}}{2 \varepsilon \ell} \int_{0}^{\infty} d k e^{-\frac{k^{2}}{2}} J_{0}(k q \ell) e^{-k d / \ell}
$$

$\left(J_{0}(q)\right.$ is the Bessel function). At small $Q$ Eq. (15) is reduced to $E_{\mathrm{mf}}=S\left(\right.$ const $\left.+\rho_{s 0} Q^{2} / 2\right)$, where

$$
\rho_{s 0}=\sin ^{2} \theta_{0} \frac{e^{2}}{16 \pi \varepsilon \ell}\left[\sqrt{\frac{\pi}{2}} \exp \left(\frac{d^{2}}{2 \ell^{2}}\right) \operatorname{erfc}\left(\frac{d}{\sqrt{2} \ell}\right)\left(1+\frac{d^{2}}{\ell^{2}}\right)-\frac{d}{\ell}\right]
$$

is the mean-field value of the superfluid stiffness (we take into account that $Q=\nabla \varphi$ ). At finite temperatures the excitations reduce this quantity. Therefore, the superfluid stiffness $\rho_{s}$ depends on temperature.

Since the gas of electron-hole pairs in bilayers is a two-dimensional one, the transition into the superfluid state is the Berezinskii-Kosterlitz-Thouless (BKT) transition. The critical temperature of the BKT transition is given by the equation

$$
T_{c}=\frac{\pi}{2} \rho_{s}\left(T_{c}\right)
$$

To obtain the dependence $\rho_{s}(T)$ one should find the spectrum of elementary excitations. Extending the approach [17, 18] to the general case of an arbitrary angle between the wave vector $\mathbf{q}$ and $\nabla \varphi$ we arrive at the following expression for the energy of excitations

$$
E(\mathbf{q})=\sqrt{\varepsilon_{\mathbf{q}, Q}\left(\varepsilon_{\mathbf{q}, Q}+2 \gamma_{\mathbf{q}, Q} \sin ^{2} \theta_{0}\right)}+\cos \theta_{0} v_{\mathbf{q}, Q},
$$


where

$$
\begin{gathered}
\varepsilon_{\mathbf{q}, Q}=2 F_{D}(Q)-F_{D}(|\mathbf{q}+Q \hat{x}|)-F_{D}(|\mathbf{q}-Q \hat{x}|), \\
v_{\mathbf{q}, Q}=F_{D}(|\mathbf{q}+Q \hat{x}|)-F_{D}(|\mathbf{q}-Q \hat{x}|), \\
\gamma_{\mathbf{q}, Q}=\left[H(\mathbf{q}, Q)-F_{S}(q)+\frac{F_{D}(|\mathbf{q}+Q \hat{x}|)+F_{D}(|\mathbf{q}-Q \hat{x}|)}{2}\right]
\end{gathered}
$$

with

$$
\mathscr{H}(\mathbf{q}, Q)=\frac{e^{2}}{2 \varepsilon \ell^{2}} e^{-\frac{q^{2} \ell^{2}}{2}} \frac{1-e^{-d q} \cos \left(q_{y} Q \ell^{2}\right)}{q},
$$

and

$$
F_{S}(q)=\frac{e^{2}}{2 \varepsilon \ell} \int_{0}^{\infty} d k e^{-\frac{k^{2}}{2}} J_{0}(k q \ell)
$$

( $\hat{x}$ is the unit vector in $x$ direction). Having the spectrum of elementary excitations one can compute the free energy

$$
F=E_{\mathrm{mf}}+T \sum_{\mathbf{q}} \ln \left(1-\exp \left(-\frac{E(\mathbf{q})}{T}\right)\right)
$$

and the density of the superfluid current

$$
j_{s}=\frac{1}{S \hbar} \frac{\partial F}{\partial Q} .
$$

On the other hand, at $Q \rightarrow 0$ the superfluid current is connected with the superfluid stiffness by the relation

$$
j_{s}=\frac{\rho_{s}(T)}{\hbar} Q .
$$

Using (24)-(26), we obtain the following expression for the superfluid stiffness

$$
\rho_{s}(T)=\rho_{s 0}+\frac{1}{S} \lim _{Q \rightarrow 0} \frac{1}{Q} \sum_{\mathbf{q}} \frac{\partial E(\mathbf{q})}{\partial Q} N_{B}(E(\mathbf{q})),
$$

where $N_{B}(E)=[\exp (E / T)-1]^{-1}$ is the Bose distribution function. For the spectrum $E(\mathbf{q})=E_{0}(q)+\hbar \mathbf{q v}$ (where $\mathbf{v}$ is the superfluid velocity, and $E_{0}(q)$ is the spectrum at $\mathbf{v}=0$ ) the temperature part of Eq. (28) yields the standard expression for the normal density [19]. The present situation differs from [19] because the dependence of the spectrum on the superfluid velocity $\mathbf{v}=\hbar Q / M\left(M=\hbar^{2} \sin ^{2} \theta_{0} / 8 \pi \rho_{s 0} \ell^{2}\right.$ is the magnetic mass of the electron-hole pair $)$ is more complicate.

The nature of such a difference is the following. In certain sense, superfluidity of electron-hole pairs in the bilayer can be considered as a kind of a counterflow superfluidity [20]. Indeed, due to the Coulomb interaction electrons from the top layer are coupled with holes from the bottom layer as well as holes from the top layer are coupled with electrons from bottom layer. In other words, one can say about two species of bosons of different polarization. In such a two-specie system only a counterflow motion of species is possible. In the limit of low mangetoexciton density the second specie can be considered as an inert background and the system behaves as a one-component gas of interacting Bose particles. But at zero imbalance of the active component we have two equivalent species that move in opposite directions with the same velocities. In particular, it results in that the last term in (19) goes to zero at $\theta_{0}=\pi / 2$. The counterflow superfluidity is a special kind of the two-component superfluidity. In similarity with the spectrum (19), the spectrum of elementary excitations of two-component superfluid system demonstrates a complicate dependence on the superfluid velocities (see, for instance, [21]).

Let us describe the behavior of the spectrum (19) at different $d / \ell$ and $Q=0$. At $d \rightarrow 0$ the spectrum reduces to the quadratic one $E(q)=\hbar^{2} q^{2} / 2 M$ (for small $q$ ). According to the Landau criterium of superfluidity it means the absence of superfluidity. At $d \approx \ell$ the roton-like minimum emerges at the dependence $E(q)$. The depth of this minimum increases under the increase of $d / \ell$ and at some critical $d=d_{c}$ the minimum touches the $q$ axis. Such a behavior of the spectrum means that at $d>d_{c}$ the system becomes instable with respect to a formation of the charge density wave. We imply the superfluidity does not survive in the charge density wave state. At $\theta_{0}=\pi / 2$ (that corresponds to zero imbalance of the active component) $d_{c} \approx 1.175 \ell$. One can expect that at $d \rightarrow 0$ and $d \rightarrow d_{c}$ the superfluid stiffness is suppressed, that results in a lowering of the critical temperature. 


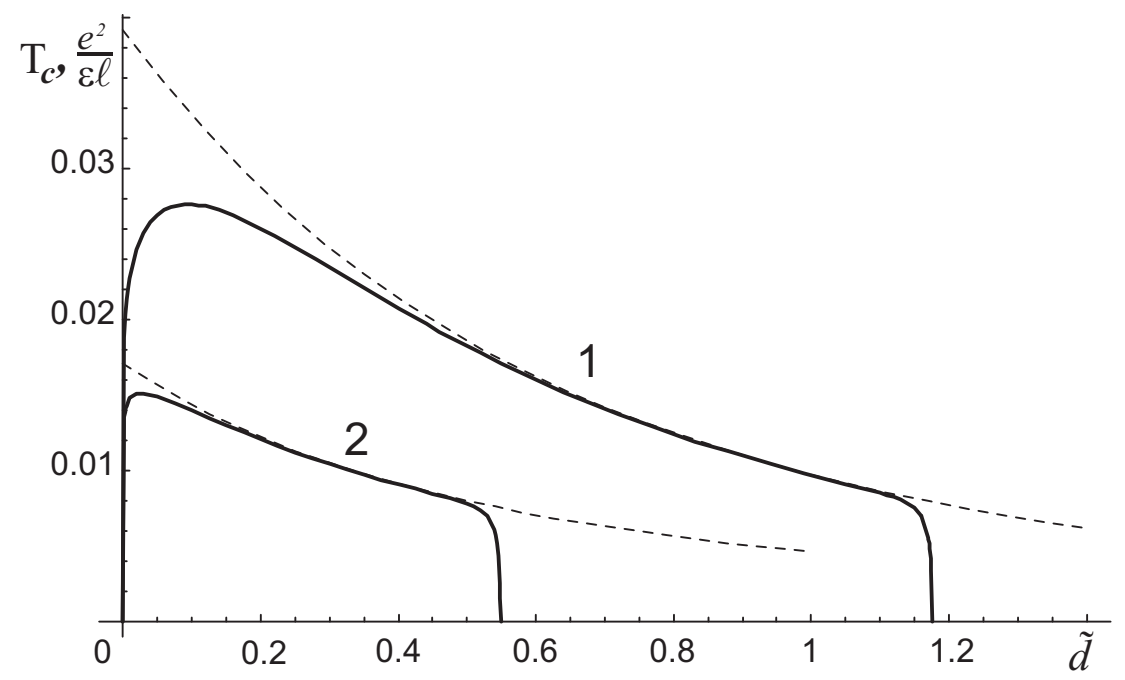

FIGURE 2. The dependence of the critical temperature on the interlayer distance $(\tilde{d}=d / \ell) ; 1$ - coupling in the zeroth Landau level, 2- coupling in the $N= \pm 1$ Landau levels. The quantities $\pi \rho_{s 0} / 2$ are shown by dashed lines.

The critical temperature obtained from Eq. (18) for the case of zero imbalance $\left(\theta_{0}=\pi / 2\right)$ is shown in Fig. 2. One can see that, indeed, at $d \rightarrow 0$ and $d \rightarrow d_{c}$ the critical temperature goes to zero. We also find that at intermediate $d$ the critical temperature can be evaluated with a good accuracy from the mean-field superfluid stiffness. Since the stiffness is proportional to $\sin ^{2} \theta_{0}=1-4 \tilde{v}^{2}$, the highest critical temperatures can be reached at zero imbalance of the active component (or at the filling factors of the zeroth Landau level $v_{1}=3 / 2, v_{2}=5 / 2$ and $v_{1}=1 / 2, v_{2}=7 / 2$ ).

The approach presented can be easily generalized for the case where electrons in the $+N$ Landau level are coupled with holes in the $-N$ level. This situation can be realized at large external gate voltage $e V>2 \sqrt{2} \hbar v_{F} / \ell$. To obtain the critical temperature in the latter case one should take into account the additional factor $f_{N}(q \ell)=\left[L_{N}\left(q^{2} \ell^{2} / 2\right)+\right.$ $\left.L_{N-1}\left(q^{2} \ell^{2} / 2\right)\right] / 2$ in the electron density operator $[8)\left(L_{N}(x)\right.$ are the Laguerre polynomials). Respectively, the factor $f_{N}^{2}(k)$ appears under the integrals in (16) and (24), and the factor $f_{N}^{2}(q \ell)$ should be added into the definition (23). Here we consider the case of the \pm 1 active level. The mean-field superfluid stiffness in this case reads as

$$
\rho_{s 0}=\sin ^{2} \theta_{0} \frac{e^{2}}{16 \pi \varepsilon \ell}\left[\sqrt{\frac{\pi}{2}} e^{\frac{d^{2}}{\ell^{2}}} \operatorname{erfc}\left(\frac{d}{\sqrt{2} \ell}\right) \frac{7+13(d / \ell)^{2}+7(d / \ell)^{4}+(d / \ell)^{6}}{16}-\frac{d\left(3 \ell^{2}+d^{2}\right)^{2}}{16 \ell^{3}}\right] .
$$

At all $d / \ell$ the superfluid stiffness for the \pm 1 Landau levels is smaller than one for the zeroth level (at the same imbalance of the active component). For the \pm 1 active levels the critical interlayer distance is $d_{c} \approx 0.549 \ell$. Using the procedure, described above, we compute the critical temperature. The result is shown in Fig. 2

\section{DISCUSSION}

We conclude that for the gas of magnetoexcitons in a bilayer graphene system the maximum critical temperature of the superfluid transition can be achieved for the coupling of electrons and holes in the zeroth Landau level and at special values of the imbalance of filling factors of the zeroth level $\left(v_{1}-v_{2}= \pm 1\right.$ and $\left.v_{1}-v_{2}= \pm 3\right)$.

For the formation of magnetoexcitons the Coulomb energy should be much smaller than the energy distance between an active and the nearest passive level. The same condition allows to neglect the effect of screening on the critical temperature. Indeed, screening can be taken into account by a substitution a $q$-dependent dielectric function $\varepsilon(q)$ instead of the dielectric constant $\varepsilon$ into the Fourier components of the Coulomb potential (see, for instance, [22]). In the case considered here the difference $\varepsilon(q)-\varepsilon$ is small by the parameter $E_{c} /\left(E_{1}-E_{0}\right)$.

For the graphene system the inequality $E_{c} \ll E_{1}-E_{0}$ is just the condition on the dielectric constant $\varepsilon \gg \varepsilon_{c} \approx 1.5$. At large $\varepsilon$ this inequality is fulfilled at all values of magnetic fields (of course, it is implied that the temperature is much smaller than $E_{1}-E_{0}$ ). The situation differs significantly from one that takes place in quantum Hall bilayers in $\mathrm{GaAs}$ heterostructures. For the carriers with the quadratic dispersion the energy distance between the Landau levels is 


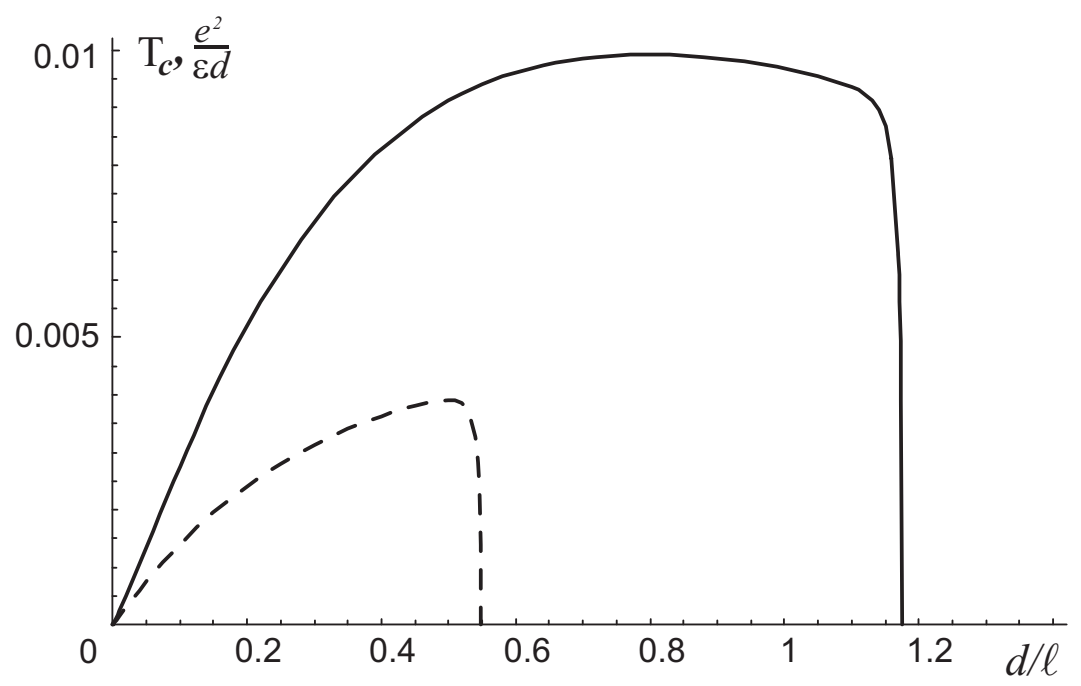

FIGURE 3. The dependence of the critical temperature on the inverse magnetic length at fixed $d$. Solid curve - magnetoexcitons in the zeroth Landau level, dashed curve - magnetoexcitons in the $N= \pm 1$ levels.

proportional to $\mathrm{B}\left(\omega_{c}=e B / m_{*} c\right.$, where $m_{*}$ is the effective mass) and the condition $E_{c}<\hbar \omega_{c}$ is fulfilled only at large magnetic fields ( $B \gtrsim 10 \mathrm{~T}$ for GaAs). The graphene bilayers require much lower $B$.

It is interesting to analyze how the critical temperature depends on $B$. Let we have a bilayer system with a given interlayer distance $d$. We vary magnetic field adjusting simultaneously the gate voltage (to keep the imbalance close to the optimal value) and try to achieve the highest critical temperature. Presenting the dependence of Fig. 2] in $e^{2} / \varepsilon d$ units we obtain (Fig. 3) the dependence of $T_{c}$ on $\ell^{-1}$ ( or on $B^{1 / 2}$ ). One can see that the critical temperature is restricted from above by $T_{c, \max } \approx 0.01 e^{2} / \varepsilon d$, and the maximum is reached for $\ell$ in the interval $[d \div 2 d]$. To achieve the critical temperature $T_{c}=1 \mathrm{~K}$ we may take the bilayer with the interlayer distance $d$ as large as $400 \AA$ (for $\varepsilon=3.9\left(\mathrm{SiO}_{2}\right)$ ) and apply comparatively low magnetic fields $B \gtrsim 0.4 \mathrm{~T}$. At the same time, our estimate shows that extremely high magnetic fields are required if we want to realize a superfluid state of magnetoexcitons at high temperatures. For instance, for $d \approx 10 \AA$ we have $T_{c, \max } \approx 40 \mathrm{~K}$, but the magnetic field should be $B>600 \mathrm{~T}$.

There is another reason that makes the case of low magnetic field important. Low fields mean large critical interlayer distances. As was shown in [23, 24], in the bilayer system the genuine superconductivity is reached in the limit of zero interlayer tunneling 11 If the systems with a finite tunneling is used for the transmission of the current from the source to the load (Fig. 11), the dissipation is nonzero. The dissipation is connected with that the state becomes nonstationary at nonzero difference of electrochemical potentials between the layers. This difference is required to provide the current in the load circuit. The power of losses is proportional to the square amplitude of the interlayer tunneling [23, 24]. A partial solution of this problem was proposed in [26]. In the setup, considered in [26], a stationary state with moving electron-hole pairs can be realized, but, regrettable, the setup [26] (and any other setup based on the same idea [24]) cannot be used for the transmission the current from the source to the load. The amplitude of tunneling decreases exponentially with the increase of the interlayer distance, and for $d \approx 400 \AA$ the effect of tunneling can be completely neglected.

\section{REFERENCES}

1. J. P. Eisenstein, and A. H. MacDonald, Nature 432, 691-694 (2004).

2. D. Snoke, Science 298, 1368 Ú1372 (2002).

\footnotetext{
${ }^{1}$ Strictly speaking, dissipation in 2D superfluid systems remains nonzero at all temperatures [25]. In the presence of a finite superfluid velocity, vortex pairs can unbind even below $T_{c}$. Although this process leads to the decay of any finite persistent current, the decay rate vanishes at zero velocity below $T_{c}$. With reference to the quantum Hall bilayers it was discussed in [13, 17]. At $T \ll T_{c}$ and for the current much lower than the mean-field critical current [17, 18] the decay rate is exponentially small and one can say about genuine superconductivity
} 
3. L. V. Butov, Solid State Commun. 127, 89 Û98 (2003).

4. Yu. E. Lozovik, and V. I. Yudson, Zh. Eksp. Teor. Fiz. 71, 738 Ü753 (1976); Sov. Phys. JETP 44, 389 Ü397 (1976).

5. S. I. Shevchenko, Fiz. Nizk. Temp. 2, 505-511 (1976); Sov. J. Low-Temp. Phys. 2, 251 Ü256 (1976).

6. K. S. Novoselov, A. K. Geim, S. V. Morozov, D. Jiang, Y. Zhang, S. V. Dubonos, I. V. Grigorieva, and A. A. Firsov, Science 306, 666-669 (2004).

7. K. S. Novoselov, A. K. Geim, S. V. Morozov, D. Jiang, M. I. Katsnelson, I. V. Grigorieva, S. V. Dubonos, and A. A. Firsov, Nature 438, 197-200 (2005).

8. H. Min, R. Bistritzer, J.-J. Su, and A. H. MacDonald, Phys. Rev. B78, 121401 (2008).

9. Yu. E. Lozovik and A. A. Sokolik, Pisma v ZhETF 87 61-65, (2008); JETP Letters 87 55-59, (2008).

10. M. Yu. Kharitonov and K. B. Efetov, Phys. Rev. B78, 241401 (2008).

11. O. L. Berman, Yu. E. Lozovik, and G. Gumbs, Phys. Rev. B77, 155433 (2008).

12. Yu. E. Lozovik, S. P. Merkulova, A. A. Sokolik, Usp. Fiz. Nauk. 187, 757-776 (2008); Phys.-Usp. 51, 727-744 (2008)

13. K. Moon, H. Mori, K. Yang, S. M. Girvin, A. H. MacDonald, L. Zheng, D. Yoshioka, and S. C. Zhang, Phys. Rev. B51, 5138-5170 (1995).

14. A. H. Castro Neto, F. Guinea, N. M. Peres, K. S. Novoselov, and A. K. Geim, Rev. Mod. Phys. 81, $109-162$ (2009).

15. S. I. Shevchenko, D. V. Fil, A. A. Yakovleva, Fiz. Nizk. Temp. 30, 431-440 (2004); Low Temp. Phys. 30, 321 Ú327 (2004).

16. A. H. MacDonald, R. Rajaraman, and T. Jungwirth, Phys. Rev. B60, 8817-8826 (1999).

17. M. Abolfath, A. H. MacDonald, and L. Radzihovsky, Phys. Rev. B68, 155318 (2003).

18. L. Yu. Kravchenko, D. V. Fil, J. Phys.: Condens. Matter 20, 325235 (2008).

19. E. M. Lifshitz and L. P. Pitaevskii, Statistical physics : pt. 2: theory of the condensed state, Pergamon Press, UK, 1980,387 $\mathrm{pp}$

20. A. B. Kuklov and B. V. Svistunov, Phys. Rev. Lett. 90, 100401 (2003).

21. L. Y. Kravchenko, D. V. Fil, J. Low Temp. Phys. 155, 219-234 (2009).

22. D. V. Fil, J. Phys.: Condens. Matter 13, 11633-11639 (2001).

23. D. V. Fil and S. I. Shevchenko, Fiz. Nizk. Temp. 33, 1023-1026 (2007); Low Temp. Phys. 33, 780 Ü782 (2007).

24. J. J. Su and A. H. MacDonald, Nature Physics 4, 799-802 (2008).

25. V. Ambegaokar, B. I. Halperin, D. R. Nelson and E. D. Siggia, Phys. Rev. Lett. 40, 783-786 (1978).

26. D. V. Fil and S. I. Shevchenko, J. Phys.: Condens. Matter 21, 215701 (2009). 